\title{
DIMENSIONALITY AND THE DUALS OF CERTAIN LOCALLY COMPACT GROUPS ${ }^{1}$
}

\section{SCHOCHETMAN}

ABSTRACT. Let $G$ be a separable locally compact group containing a closed normal subgroup which is type I and regularly embedded. The elements of the dual space $\hat{G}$ are known to be induced from certain closed subgroups. In this article we first determine the kernel of an induced representation and then give necessary and sufficient conditions for $G$ to be maximally almost periodic. These results are then applied to the collection of compact extensions of abelian groups.

Introduction. In [6, Theorem 8.4] G. W. Mackey described the structure of the irreducible representations of a separable locally compact group $G$ having a closed normal subgroup $N$ which is type I and regularly embedded. These representations are induced from certain closed subgroups. Our main purpose here is to give necessary and sufficient conditions for the above group $G$ to have sufficiently many finite dimensional irreducible representations to separate its points, i.e. for $G$ to be maximally almost periodic. To accomplish this we must first determine the kernel of an induced representation. This is done in $\$ 2$.

In $\$ 3$ we give a classification of the collection of compact extensions of abelian groups based on the previous results and study a particular example.

1. Preliminaries. Throughout this paper, all groups will be separable and locally compact (with identities denoted by $e$ ) and all representations will be strongly continuous, unitary and of countable dimension. We will not distinguish between a representation and its (unitary) equivalence class. If $\sigma$ is a multiplier for $G[6$, p. 267] then $(G, \sigma)^{\wedge}$ will denote the $\sigma$-dual of $G$, i.e. the equivalence classes of irreducible $\sigma$-representations of $G$ (see $[6$, p. 272]). $\hat{G}$ will be the usual dual of $G$ corresponding to trivial $\sigma$.

Received by the editors October 29, 1969 and, in revised form, February 13, 1970.

AMS 1969 subject classifications. Primary 2265, 2260; Secondary 2220.

Key words and phrases. Locally compact group, normal subgroup, group extension, dual space, finite dimensional representation, irreducible representation, kernel of a representation, induced representation, maximally almost periodic.

1 This article constitutes a portion of the author's Ph.D. Dissertation written at the University of Maryland under the direction of Professor A. Kleppner. 
Let $G$ be a group, $N$ a closed normal subgroup which is type $I$ and regularly embedded $[6$, p. 302]. We then have a mapping $G \times \hat{N} \rightarrow \hat{N}$ given by $(y, L) \rightarrow L^{y}$, where $L_{x}^{\nu}=L_{y x y^{-1}}$, for $x \in N$. For each $L \in \hat{N}$ we let $\theta(L)$ be the orbit $\left\{L^{y}: y \in G\right\}$. The orbits partition $\hat{N}$ and give rise to the orbit space $\hat{N} / G$. If $L \in \hat{N}$, then $H_{L}=\left\{y \in G: L^{y}=L\right\}$ is a closed subgroup of $G$ containing $N$ [6, Theorem 7.5] which we call the stability subgroup of $L$. Let $\pi_{L}: H_{L} \rightarrow H_{L} / N$ be the corresponding canonical homomorphism. For each $L \in \hat{N}$, let $\mathfrak{N}_{L}$ denote the set of $T$ in $\hat{H}_{L}$ such that the restriction $T \mid N$ is a multiple of $L$. If $\sigma_{L}$ is a multiplier for $H_{L} / N$, then $\sigma_{L} \pi_{L}$ is a multiplier for $H_{L}$ as is its inverse $\left(\sigma_{L} \pi_{L}\right)^{-1}$. Applying Theorem 8.4 of [6] (see also [2, Proposition 1]) for the case of the trivial multiplier we have

MACKEY'S TheOREM. Let the notation be as above. There exists an onto mapping $p: \hat{G} \rightarrow \hat{N} / G$ such that if $\hat{G}_{\theta}=p^{-1}(\theta)$, for $\theta \in \hat{N} / G$, then the mapping $T \rightarrow U^{T}$ (induced representation of $G$ ) is a bijection between $\mathfrak{T}_{L}$ and $\hat{G}_{\theta}$, where $L$ is any element of the orbit $\theta$. The mapping $p$ is given by $p\left(U^{T}\right)=\theta$. Furthermore, there exists a unique (up to similarity [6, p. 269]) multiplier $\sigma_{L}$ for $H_{L} / N$ such that $L$ extends to a $\left(\sigma_{L} \pi_{L}\right)^{-1}{ }_{-}$ representation $L^{\prime}$ of $H_{L}$ and the mapping $M \rightarrow L^{\prime} \otimes M \pi_{L}$ is a bijection between $\left(H_{L} / N, \sigma_{L}\right) \wedge$ and $\mathfrak{M}_{L}$.

Observe that $p$ is onto as a consequence of the discussion on pp. 27-28 of [1]. Note also that if $L$ is the identity element of $\hat{N}$, then $H_{L}=G$ and $\sigma_{L}$ is trivial so that $\left(H_{L} / N, \sigma_{L}\right)^{\wedge}=(G / N)^{\wedge}$.

Definition 1.1. Let $G$ be a group. If $\sigma$ is a multiplier for $G$, then $(G, \sigma)^{\wedge}$ has property (FDR) if every element of $(G, \sigma)^{\wedge}$ is finite dimensional. When $\sigma$ is trivial we will delete it. [FDR] will denote the collection of such groups (these are denoted by [Moore] in [9]).

If $H$ is a closed subgroup of $G, \mu$ a quasi-invariant measure on $G / H$ and $T$ a representation of $H$ in the Hilbert space $\mathcal{F}(T)$, then the Hilbert space of $U^{T}$ is well known to be unitarily equivalent to $L^{2}(G / H, \mu, \mathfrak{F}(T))$. Hence, we have

LemMa 1.2. Let $G$ be a group, $H$ a closed subgroup and $T$ a representation of $H$. Then $U^{T}$ is finite dimensional if and only if $T$ is finite dimensional and $H$ is of finite index in $G$ (and hence open).

As a direct consequence of this lemma and Mackey's Theorem we can prove

THEOREM 1.3. Let $G$ be a group and $N$ a closed normal subgroup which is type I and regularly embedded. The remaining notation is as above. Then $G$ is in [FDR] if and only if 
(i) $N$ is in [FDR],

(ii) each $\left(H_{L} / N, \sigma_{L}\right)^{\wedge}$ has property (FDR), and

(iii) each $H_{L}$ is of finite index in $G$, for $L \in \hat{N}$.

2. Main result. Recall that the group $G$ is called maximally almost periodic (MAP) if for each $y \in G, y \neq e$, there exists finite dimensional $V$ in $\hat{G}$ such that $y \notin \operatorname{ker}(V)$. We denote the collection of such groups by [MAP]. The next lemma is the key to determining when $G$ is in [MAP].

Lemma 2.1. Let $G$ be a group, $H$ a closed subgroup and $T$ a representation of $H$. Let $\Delta_{G}$ and $\Delta_{H}$ be the modular functions for $G$ and $H$ respectively. Define $\delta(z)=\Delta_{H}(z) / \Delta_{G}(z)$, for $z \in H$. Then $\delta$ is a continuous homomorphism of $H$ into the multiplicative group of positive real numbers and

$$
\operatorname{ker}\left(U^{T}\right)=\left(\bigcap_{y \in G} y \operatorname{ker}(T) y^{-1}\right) \cap\left(\bigcap_{y \in G} y \operatorname{ker}(\delta) y^{-1}\right) .
$$

Proof. The first assertion is obvious. Let $F$ denote the subspace of $\mathfrak{F}\left(U^{T}\right)$ of continuous "functions" with compact support modulo $H$. Then $F$ is dense in $\mathfrak{F}\left(U^{T}\right)$ [7, Lemma 3.5]. Let $\rho$ be as in Lemma 1.4 of [7] ( $\rho$ may be assumed continuous). Suppose first that $x \in$ $\operatorname{ker}\left(U^{T}\right)$. Then from the definition of $U^{T}[7, \mathrm{p} .107]$ we have

$$
U_{x}^{T} f(y)=(\rho(y x) / \rho(y))^{1 / 2} f(y x)=f(y),
$$

for all $f \in F$ and $y \in G$. If $y x$ and $y$ belong to different $H$-cosets, we can find $f$ in $F$ such that $f(y) \neq f(y x)=0$ (this follows from Lemma $3.4(d)$ of [7]), i.e. $F$ separates points in $G$ modulo $H$. Thus, $y x y^{-1}$ is in $H$ for all $y$ in $G$. Consequently,

$$
\begin{aligned}
f(y) & =\left(\rho\left(y x y^{-1} y\right) / \rho(y)\right)^{1 / 2} f\left(y x y^{-1} y\right) \\
& =\delta\left(y x y^{-1}\right) T_{y x y^{-1}} f(y), \quad f \in F, y \in G .
\end{aligned}
$$

Since $\{f(y): f \in F\}$ is dense in $\mathfrak{F}(T)$ [7, Lemma 3.2], we see that for each $y \in G$, the operator $\delta\left(y x y^{-1}\right) T_{y x y^{-1}}$ must be the identity on $\mathfrak{H C}(T)$. This is true only if $y x y^{-1} \in \operatorname{ker}\left(U^{T}\right) \cap \operatorname{ker}(\delta)$. Thus we have the forward inclusion. The reverse argument gives the other inclusion.

Definition 2.2. Let $L \in \hat{N}$. Then $L$ is finitely inducible if there exists $T$ in $\mathfrak{T}_{L}$ such that $U^{T}$ is finite dimensional, i.e. if there exists finite dimensional $V$ in $\hat{G}$ such that $L$ belongs to the orbit $p(V)$.

From Mackey's Theorem and Theorem 1.3 we have 
LEMMA 2.3. L is finitely inducible if and only if

(i) L is finite dimensional,

(ii) there exists $M$ in $\left(H_{L} / N, \sigma_{L}\right)^{\wedge}$ which is finite dimensional and

(iii) $H_{L}$ is of finite index in $G$.

Note that the identity element of $\hat{N}$ is always finitely inducible; however, it may be the only one. Denote the set of finitely inducible elements in $\hat{N}$ by $\hat{N}_{f}$. For each $L \in \hat{N}_{f}$, let

$$
\begin{aligned}
\mathscr{F}_{L} & =\left\{T \in \mathfrak{N}_{L}: T \text { is finite dimensional }\right\} \\
& =\left\{L^{\prime} \otimes M \pi_{L}: M \text { is a finite dimensional element of }\left(H_{L} / N, \sigma_{L}\right)^{\wedge}\right\} .
\end{aligned}
$$

Furthermore, define $\mathcal{F}=\bigcup_{L \in \hat{N}_{f}} \mathcal{F}_{L}$ and $K=\bigcap_{T \in \mathfrak{F}} \operatorname{ker}\left(U^{T}\right)$.

REMARKs. (1) If $T \in \mathcal{F}$, then $T$ is induced from an open subgroup in which case the corresponding $\delta$ (Lemma 2.1 ) is identically 1 . Hence,

$$
K=\bigcap_{T \in \mathcal{F}} \bigcap_{y \in G} y \operatorname{ker}(T) y^{-1},
$$

so that $K$ is a closed normal subgroup of $G$ contained in each $H_{L}$, $L \in \hat{N}_{f}$.

(2) The set of finite dimensional elements of $\hat{G}$ is precisely $\left\{U^{T}: T \in \mathcal{F}\right\}$; hence, $G \in[\mathrm{MAP}]$ if and only if $K=\{e\}$.

(3) Let $L \in \hat{N}_{f}$ and let $M \in\left(H_{L} / N, \sigma_{L}\right)^{\wedge}$ be as in Lemma 2.3. If $y \in G$, then $L^{y} \in \hat{N}$ and $H_{L^{y}}=y^{-1} H_{L} y$. It follows from Mackey's Theorem that there exists a unique element in $\left(H_{L^{y}} / N, \sigma_{L^{\nu}}\right)^{\wedge}$, which we will call $M^{v}$, such that $U^{T}=U^{S}$, where $T=L^{\prime} \otimes M \pi_{L}$ and $S$ $=\left(L^{\nu}\right)^{\prime} \otimes M^{y} \pi_{L^{\nu}}$. Necessarily, $M^{y}$ is finite dimensional. Since $H_{L}$ is of finite index in $G$, so is $H_{L^{\nu}}$. Furthermore, $L^{\nu}$ is finite dimensional so that $L_{y} \in \hat{N}_{f}$, i.e. $\hat{N}_{f}$ is a union of orbits in $\hat{N}$.

Proposition 2.4. $K=\bigcap_{T \in \mathcal{F}} \operatorname{ker}(T)$.

Proof. Let $T \in \mathcal{F}, y \in G$. Then $T \in \mathcal{F}_{L}$, for some $L \in \hat{N}_{f}$. For each $z \in y^{-1} H_{L} y$, define $T_{z}^{y}=T_{y z y^{-1}}$. Then it is easy to see that $T^{y}$ is an irreducible representation of $H_{L^{y}}=y^{-1} H_{L} y$ and $T^{\nu} \mid N$ is a multiple of $L^{\nu}$, i.e. $T^{\nu} \in \mathscr{T}_{L^{\nu}}$. Hence, $T^{\nu} \in \mathcal{F}_{L^{\nu}} \subseteq \mathcal{F}$ (note that $L \in \hat{N}_{f}$ implies $\left.L^{\nu} \in \hat{N}_{f}\right)$. It is also easy to see that $\operatorname{ker}\left(T^{v}\right)=y^{-1} \operatorname{ker}(T) y$, which completes the proof.

In general there are two kinds of points in $K$-those in $N$ and those not in $N$. From the previous proposition, separating a point of $G \sim N$ (set difference) from $e$ by a finite dimensional element of $\hat{G}$ is the task of the family $\mathcal{F}$. However, separating a point in $N$ from $e$ is possible precisely when it can be accomplished by an element of $\hat{N}_{f}$. This follows from Proposition 2.4 together with the fact that for each $L \in \hat{N}$ the elements of $\Re_{L}$ restrict on $N$ to multiples of $L$. 
Definition 2.5. The set $\hat{N}_{f}$ is separating if $\bigcap_{L \in \hat{N}_{f}} \operatorname{ker}(L)=\{e\}$.

Note that if $\hat{N}_{f}$ is separating then $N \in[\mathrm{MAP}]$. As a consequence of the preceding we have our main result:

TheOREM 2.6. Let $G$ and $N$ be as in Mackey's Theorem. Then the following are equivalent:

(i) $G \in[\mathrm{MAP}]$.

(ii) $\bigcap_{T \in \mathcal{F}} \operatorname{ker}(T)=\{e\}$.

(iii) $\bigcap_{T \in \mathcal{F}} \operatorname{ker}(T) \subseteq N$ and $\hat{N}_{f}$ is separating.

Let $\mathscr{L}$ be a subset of $\hat{N}_{f}$ which is also a union of orbits. Let $\mathcal{F}_{\mathscr{L}}=\bigcup_{L \in \mathcal{L}} \mathcal{F}_{\mathcal{L}}$ and define $\mathscr{L}$ to be separating by making the obvious changes in the corresponding definition of $\hat{N}_{f}$. Clearly, $\hat{N}_{f}$ is separating if and only if $\mathscr{L}$ is separating for some $\mathscr{L}$ as above.

COROLLARY 1. In the theorem, $\mathcal{F}$ may be replaced by $\mathcal{F}_{\mathfrak{L}}$ and $\hat{N}_{f}$ by $\mathfrak{L}$ for $\mathfrak{L}$ as above.

Corollary 2. If $G / N \in[\mathrm{MAP}]$, then $G \in[\mathrm{MAP}]$ if and only if $\hat{N}_{f}$ is separating.

Proof. If $I$ denotes the identity element of $\hat{N}$, then in this case $\bigcap_{T \in \mathcal{F}_{I}} \operatorname{ker}(T)=N$ so that $\bigcap_{T \in \mathcal{F}} \operatorname{ker}(T) \subseteq N$ automatically.

3. Compact extensions of abelian groups. Throughout this section we will consider the class of separable locally compact groups $G$ having a closed normal abelian subgroup $A$ (depending on $G$ ) for which $G / A$ is compact. Denote this collection by [CA]. Observe that for given $G$ in $[\mathrm{CA}], A$ need not be unique. Recall that abelian groups are type $I$.

LemMA 3.1. If $G \in[\mathrm{CA}]$ then $A$ is regularly embedded in $G$.

Proof. Since the action of $G$ on $\hat{A}$ is constant on the cosets of $A$, we obtain the transformation group $G / A \times \hat{A} \rightarrow \hat{A}$, where $\hat{A}$ is locally compact Hausdorff and $G / N$ is compact. It follows that the orbit space $\hat{A} / G$ is itself Hausdorff.

Hence, we may apply all of the preceding results to the groups in [CA].

Notation. Let $[Z]=$ those $G$ in $[C A]$ for which $A$ may be chosen to be the center of $G$. $[\mathrm{FZ}]=$ the set of separable locally compact groups $G$ having a normal subgroup $N$ of finite index such that $N \in[Z]$.

Clearly, $[\mathrm{FZ}] \subseteq[\mathrm{CA}]$. The collection $[\mathrm{Z}]$ (without the separability assumption) has been studied in great detail by $\mathrm{S}$. Grosser and $\mathrm{M}$. Moskowitz who call them central groups (see [3] and [4]). 
Let us define $H=\bigcap_{\mathbf{x} \in \hat{\mathbf{A}}} H_{\mathrm{x}}$ which is a closed normal subgroup of $G$ containing $A$ (depending on the choice of $A$ ) and $S=\left\{\chi \in \hat{A}: H_{\chi}\right.$ is open\}.

\section{Lemma 3.2. (i) $H$ is the centralizer of $A$ in $G$. (ii) $H \in[Z]$.}

Proof. (i) This follows from the fact that the points of $A$ are separated by the characters in $\hat{A}$.

(ii) By (i), $A$ is contained in the center of $H$ where $H / A$ is necessarily compact.

Theorem 3.3. Let $G \in[C A]$. Then:

(i) $G \in[Z]$ if and only if for some $A$ we have $H=G$, i.e. $H_{x}=G$ for all $\chi \in \hat{A}$.

(ii) $G \in[\mathrm{FZ}]$ if and only if for some $A, H$ is open.

(iii) $G \in[\mathrm{FDR}]$ if and only if for any $A, H_{x}$ is open for all $\chi \in \hat{A}$.

(iv) $G \in[\mathrm{MAP}]$ if and only if for any $A, \cap_{x \in S} \operatorname{ker}(\chi)=\{e\}$.

We therefore have the following chain of inclusions.

$$
[\mathrm{Z}] \subseteq[\mathrm{FZ}] \subseteq[\mathrm{FDR}] \cap[\mathrm{CA}] \subseteq[\mathrm{MAP}] \cap[\mathrm{CA}] \subseteq[\mathrm{CA}]
$$

The second inclusion follows from Theorem 1.3. It can be seen from specific examples that all of these inclusions (with the possible exception of the second) are proper. To complete the picture, we define [FA] to be the set of $G$ in [CA] for which $G / A$ is finite for some $A$. Clearly, $[\mathrm{FA}] \subseteq[\mathrm{FZ}]$. The significance of the collection $[\mathrm{FA}]$ is that the dual elements of such a group are bounded in degree (this is an unpublished result of C. C. Moore). It may also be seen to be a consequence of Mackey's Theorem. Finally, it is worth noting that the forward implication of part (iv) of Theorem 3.3 (together with Lemma 3.2(i)) implies Theorem 6 of [10] for the particular case of a [CA]-group.

As a particular example, let $A$ be a direct sum of countably many copies of the integers $Z$ with the discrete topology, $Q$ a direct product of countably many copies of the cyclic group $F=\{1,-1\}$ of order two with the product topology and $G$ the semidirect product $A \cdot Q$ with the product topology and the group operation given by

$$
(a, q)(b, r)=(a+q b, q r), \quad a, b \in A, q, r \in Q,
$$

where $q b=\left(q_{i} b_{i}\right)$. This example is due to Murakami [8] and is a [CA]-group. The dual $\hat{A}$ is equal to a product of the circle group $T$ (of complex numbers of modulus 1) with itself countably many times [5, p. 365]. Hence, an element of $\hat{A}$ is of the form $\chi_{t}$, where $\ell=\left(t_{i}\right), t_{i} \in T, i=1,2, \cdots$, and $\chi_{t}(a)=\prod_{i=1}^{\infty} t_{i}^{a_{i}}, a=\left(a_{i}\right) \in A$. Note 
that this product is finite. Let $\chi_{t}$ be a nonidentity element of $\hat{A}$, i.e. $t_{i} \neq 0$, some $i$, and $H_{t}$ the corresponding stability subgroup. Let $y \in G$ and suppose $y=(b, q)$. Then $\chi_{t}^{y}(a)=\chi_{t}(q a)=\chi_{q t}(a), a \in A$, where $q t=\left(q_{i} t_{i}\right)$.

Proposition 3.4. For all $t \in \hat{A}, H_{t}=\left\{(a, q) \in G: a \in A\right.$ and $q_{i}$ is arbitrary if $t_{i}=1$ or $-1, q_{i}=1$ otherwise $\}$.

The group $G$ does not belong to [FDR]; in fact, if for each $j$ we let $t_{j}=\alpha=\cos (1)+i \sin (1)$, then $H_{t}=A$ for $t=\left(t_{j}\right)$, which is not open. However, if we fix $j$ and let $t^{j}=(1, \cdots, 1, \alpha, 1, \cdots)$ (with $\alpha$ in the $j$ th position), then

$H_{t^{i}}=A \cdot(F \times \cdots \times F \times\{1\} \times F \times \cdots) \quad(\{1\}$ in the $j$ th position $)$,

which has index two and is open. Furthermore, if $\chi_{j}=\chi_{t}{ }^{i}$ then $\chi_{j}(a)=\alpha^{a_{j}}, a \in A$, where $\alpha^{a_{j}=1}$ if and only if $a_{j}=0$. Hence, $\operatorname{ker}\left(\chi_{j}\right)$ $=\left\{a \in A: a_{j}=0\right\}$ and $\bigcap_{j=1}^{\infty} \operatorname{ker}\left(\chi_{j}\right)=\{0\}$. Therefore, $G \in[\mathrm{MAP}]$ $\sim$ [FDR] (Theorem 3.1 (iv)).

\section{REFERENCES}

1. L. Auslander and C. C. Moore, Unitary representations of solvable Lie groups, Mem. Amer. Math. Soc. No. 62 (1966). MR 34 \#7723.

2. J. M. G. Fell, $A$ new proof that nilpotent groups are $C C R$, Proc. Amer. Math. Soc. 13 (1962), 93-99. MR 24 \#A3238.

3. S. Grosser and M. Moskowitz, On central topological groups, Trans. Amer. Math. Soc. 127 (1967), 317-340. MR 35 \#292.

4. - Representation theory of central topological groups, Trans. Amer. Math. Soc. 129 (1967), 361-390. MR 37 \#5327.

5. E. Hewitt and K. A. Ross, Abstract harmonic analysis. Vol. I: Structure of topological groups. Integration theory, group representations, Die Grundlehren der math. Wissenschaften, Band 115, Springer-Verlag, Berlin and New York, 1963. MR $28 \# 158$.

6. G. W. Mackey, Unitary representations of group extensions. I, Acta Math. 99 (1958), 265-311. MR 20 \#489.

7. - Induced representations of locally compact groups. I, Ann. of Math. (2) 55(1952), 101-139. MR 13, 434.

8. S. Murakami, Remarks on the structure of maximally almost periodic groups, Osaka Math. J. 2 (1950), 119-129. MR 13, 12.

9. L. C. Robertson, A note on the structure of Moore groups, Bull. Amer. Math. Soc. 75 (1969), 594-599.

10. T. W. Wilcox, On the structure of maximally almost periodic groups, Math. Scand. 23 (1968), 221-232.

University of Maryland, College Park, Maryland 20742

OAKLAND University, Rochester, Michigan 48063 